\title{
Measuring the Magnet that Measures the Muon
}

\author{
To precisely measure the magnetic moment of the muon, physicists first \\ needed to precisely measure the field produced by the 680 -ton magnet \\ that guides the muons.
}

\author{
By Michael Schirber
}

$\prod$ he basic idea of the Muon $g-2$ experiment at Fermi National Accelerator Laboratory (Fermilab), Illinois, is to detect the wobbles of microscopic magnets traveling around a 15-m-wide ring-shaped magnet. The tiny magnets are elementary particles called muons, and the wobbles reveal the magnetic strength, or moment, of the muons. The results reported today don't match up with standard model predictions, which is making the muon the talk of the town (see Viewpoint: Muon's Escalating Challenge to Standard Model). However, there's a less talked about aspect to this development, and that's the giant magnet that corrals the muons. The Muon $g-2$ scientists constantly keep tabs on this 1.45-tesla magnet using hundreds of nuclear magnetic

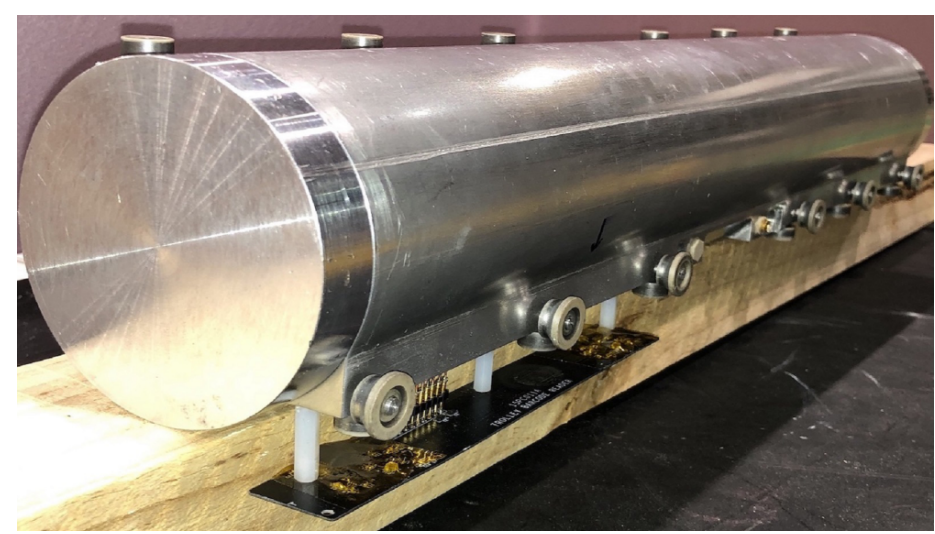

Image of the trolley carrying the nuclear magnetic resonance probes used to measure the magnetic field in the Muon $g-2$ experiment.

Credit: Argonne National Laboratory resonance (NMR) sensors, some of which ride on a small trolley that rolls around the experiment. The effort has helped bring down the uncertainties in the field measurement to 114 parts per billion-a nearly twofold improvement over the previous muon experiment, where the magnetic-moment discrepancy was first observed.

That experiment, which ran at Brookhaven National Laboratory, New York, used the same giant magnet that Muon $g-2$ is using today. The magnet was shipped 3200 miles from Brookhaven to Fermilab in 2013. The magnet's main components are a combination of iron chunks and superconducting coils that produce a vertical field inside the muon storage ring-a 45-m-long circular path tucked within the magnet's metal structure. The field steers the muons along a circular path, while also causing their magnetic moments to wobble, or precess. To obtain the muon's magnetic moment, the Muon $g-2$ Collaboration divides the frequency of this precession by the strength of the magnetic field. "The spin precession frequency is the more familiar part of the experiment," says Peter Winter from Argonne National Laboratory, Illinois. "But measuring the magnetic field is just as important."

Winter and his colleagues have developed an elaborate set of protocols for measuring the magnetic field, which they don't quantify in terms of teslas but in terms of the precession frequency of a proton exposed to the same field while sitting at the center of a spherical water sample at $34.7^{\circ} \mathrm{C}$. "It's a mouthful," admits David Kawall from the University of Massachusetts, Amherst. But this proton-in-water frequency is a commonly used standard in NMR measurements. Kawall 


\section{RESEARCH NEWS}

compares it to the metal cylinder in Paris that was-until recently-the kilogram standard. "We know how to take what our probes measure and interpret it in terms of this NMR standard," Kawall says.

One of the complications in measuring the field of the giant magnet is that it varies both in space and in time because of structural inhomogeneities and temperature variations. "If the storage ring were perfectly homogeneous, then you could just put in one probe, measure the field, and you'd be done," Kawall says. The spatial deviations around the ring are of order 14 to 17 parts per million-which isn't terrible for a giant iron magnet, he says. In fact, the deviations are 3 times smaller than for the Brookhaven experiment, thanks in part to a meticulous "shimming" process, in which 8000 hand-cut strips of iron foil were glued onto the magnet structure in 2016. The foil strips leveled out the field-like sheets of paper placed under the legs of a wobbly table. "These small pieces can make a sizable change in the magnetic field," says David Flay form Jefferson Lab in Virginia.

Even with all the adjustments made to the magnet, the researchers need a detailed map of the field. For that, they have installed an array of 378 NMR probes around the magnet ring. These fixed probes can provide continuous readings of the field, but they sit several centimeters away from the muon beam. To measure the actual field that the muons experience, Winter and his colleagues seated 17 NMR probes inside a 50-cm-long trolley. Every three days-when the muon beam is shut off-the cylindrical trolley is dragged out of a small garage and pulled around the beam path by a set of cables. Although it carries no passengers, the trolley has a full itinerary with 9000

"destinations" where it records field measurements. "The trolley can map the field at finer intervals than the fixed probes, giving us a better measurement of the field distribution distribution where the muons move." Winter says. Poking along at a speed of roughly $1 \mathrm{~cm} / \mathrm{s}$, the trolley takes about one hour to complete a one-way trip around the 45-m circumference.
The probes in the trolley, as well as the fixed ones, are 10-cm-long cylinders filled with a dab of petroleum jelly. Protons in the jelly are made to precess through the application of a radio pulse, and this precession is detected to determine the magnetic field around the probe. "We use petroleum jelly because the proton precession recovery time is faster than in water, allowing us to measure the field every 1.4 seconds," Flay explains. To convert the proton-in-jelly frequency measurement to the standard proton-in-water frequency, Flay and Kawall developed a water-based NMR probe that they station at a single stop along the trolley path. During the calibration process, the trolley moves in, takes a measurement at a well-defined position, and moves out. Then, the calibration probe executes the exact same maneuvers, and the readings are compared. This "hokey pokey dance" is repeated over and over for six hours to obtain a reliable conversion factor for each probe in the trolley.

"I think the magnetic field measurement is sometimes under-appreciated in this experiment because one might think it just involves placing a sensor somewhere," Winter says. "In reality, it's a long chain of complex measurements." The researchers continue to work on reducing the measurement uncertainties, with the goal of reaching 70 parts per billion precision for the magnetic field and 140 parts per billion for the muon magnetic moment. The experiment is a rich mix of high-energy physics, atomic physics, and beam dynamics, says Kawall, who worked on the Brookhaven experiment before joining the Fermilab effort. "It's so interesting, you could spend a whole career working on it to try and understand it," he says.

Michael Schirber is a Corresponding Editor for Physics based in Lyon, France. 